\title{
Reentrant Condensation of DNA induced by Multivalent Counterions
}

\author{
T. T. Nguyen, I. Rouzina* and B. I. Shklovskii \\ Theoretical Physics Institute, University of Minnesota, 116 Church St. Southeast, Minneapolis, Minnesota 55455 and \\ *Department of Biochemistry, University of Minnesota, 1479 Gortner Ave. St. Paul, Minnesota 55108
}

A theory of condensation and resolubilization of a dilute DNA solution with growing concentration of multivalent cations, $N$ is suggested. It is based on a new theory of screening of a macroion by multivalent cations, which shows that due to strong cation correlations at the surface of DNA the net charge of DNA changes sign at some small concentration of cations $N_{0}$. DNA condensation takes place in the vicinity of $N_{0}$, where absolute value of the DNA net charge is small and the correlation induced short range attraction dominates the Coulomb repulsion. At $N>N_{0}$ positive DNA should move in the oppisite direction in an electrophoresis experiment. From comparison of our theory with experimental values of condensation and resolubilization thresholds for DNA solution containing Spe ${ }^{4+}$, we obtain that $N_{0}=3.2 \mathrm{mM}$ and that the energy of DNA condensation per nucleotide is $0.07 k_{B} T$.

PACS numbers: 77.84.Jd, 61.20.Qg, 61.25Hq

\section{INTRODUCTION}

In the last several years there has been a revival of interest in the phenomenon of DNA condensation with multivalent cations. The reason for this interest is the general effort of the scientific community to develop effective ways of gene delivery for the rapidly growing field of genetic therapy. The DNA compaction should be fast, effective, easily reversible and should not damage the DNA double helix. All of these conditions are fulfilled in DNA condensation with multivalent cations, such as $\mathrm{CoHex}^{3+}$, naturally occurring polyamines $\mathrm{Spd}^{3+}, \mathrm{Spe}^{4+}$ and their analogs which are known to bind to DNA in the predominantly nonspecific electrostatic manner. The DNA condensates obtained this way are indeed closely packed arrays of parallel DNA strands. It was shown that the helical structure of the B-DNA is not perturbed within such condensate, and that the reaction is easily reversed by the addition of monovalent salt, or simply dilution of the solution with water. Cations with larger and more compact charge are more effective in condensing the DNA. This also suggests an electrostatic mechanism of the DNA condensation with multivalent cations. During about twenty years of research, a significant amount of information on DNA condensation has been accumulated. For long DNA, as the concentration of $Z$-valent cations grows condensation happens abruptly at some critical concentration, $N_{c}$ which depends on the charge of cations and the concentration of monovalent salt $n$. A comprehensive review of the experimental and theoretical results for $N_{c}$ can be found in Refs. 12.2.

The intensive study of the last few years revealed completely new features of DNA condensation with multivalent cations 3 6. It was discovered that when the concentration of cations grows far beyond $N_{c}$ to some new critical value $N_{d} \gg N_{c}$, DNA dissolves and returns to the solution. This reentrance condensation behaviour is schematically shown on Fig. 1. For a long DNA and small $n$ both transitions are very sharp and the ratio of
$N_{d} / N_{c}$ can be as large as $10^{4}$. Remarkably, the decondensation threshold $N_{d}$ is almost totally independent on the monovalent salt concentration, $n$. On the other hand, the condensation threshold, $N_{c}$, grows with increasing $n$.

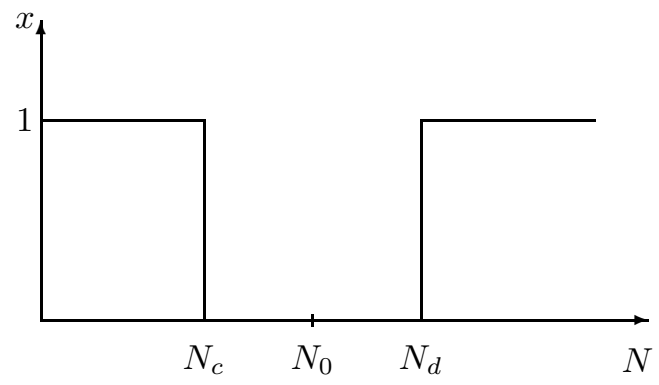

FIG. 1. Schematical illustration of the reentrant condensation. The fraction $x$ of DNA molecules in solution is plotted as a function of logarithm of the cation concentration

It has been understood for some time that, due to correlations between multivalent cations at the DNA surface 15 , two DNA molecules experience a short range attraction, which can lead to condensation. Monovalent ions are much less correlated and do not provide any attraction. Conventional explanation for the condensation threshold $N_{c}$ is that it is just the bulk concentration of $Z$-valent cations, $N=N_{r}$, at which they replace monovalent ones and begin to produce a short range attraction. However, from this point of view it is very difficult to understand why at large $N=N_{d}$, DNA molecules go back to the solution.

The above explanation also does not take into account the net charge of DNA The nonlinear PoissonBoltzmann equation predicts 18.19 a value for the net linear charge density of DNA, $\eta^{*}$, which includes the bare charge of DNA and the charge of cations bound at the very surface of DNA with energy larger than $k_{B} T$. This charge is negative and does not depend on $N$. At 
$N>N_{r}$, two situations are possible. The energy of the Coulomb repulsion can be smaller than the energy of the short range attraction. Then DNA condenses at $N=N_{r}$ but never dissolves back. If the energy of the Coulomb repulsion is larger than the energy of short range attraction, condensation does not happen at all. Both possibilities contradict experiments and therefore reentrant condensation can not be explained in the Poisson-Boltzmann aproximation.

In this paper we propose an explanation for the reentrant condensation based on a new theory of screening of macroions by multivalent cations, which emphasizes the strong correlations of multivalent cations at the surface of DNA. We explain why the condensed phase exists only within the limited range, $N_{c} \leq N \leq N_{d}$ and calculate both $N_{c}$ and $N_{d}$.

It was shown in Ref. 16, 17, that the strong repulsion between multivalent cations leads to their strong lateral correlations. The resulting strongly correlated liquid of multivalent cations at the surface of DNA has a large negative chemical potential, which describes the additional purely electrostatic binding of cations to the surface. This, in turn, leads to an exponentially small concentration of cations, $N_{0}$, above the surface. $\left(N_{0}>N_{r}\right.$, if $n$ is not unrealistically large). According to Ref. 16, 17 , when the bulk concentrations of multivalent cations, $N$, grows above $N_{r}$, the net negative charge $\eta^{*}$ decreases in absolute value and crosses zero at $N=N_{0}$. At $N>N_{0}$, the net charge becomes positive and continues to grow with $N$. This effect is called charge inversion. It is worth noting here, that the charge inversion is not a result of some specific chemosorbtioned, but rather a direct consequence of purely electrostatic interactions of multivalent cations.

At $N=N_{0}$ there is no Coulomb repulsion at all, so that the short range attraction dominates and leads to condensation. This is actually the optimal situation for condensation. It is obvious that there is a range of $N$ around $N_{0}$ where attraction still dominates and DNA condences. Condensation thresholds $N_{c}$ and $N_{d}$ then are determined by the condition that the energy of the short range attraction is equal to the energy of Coulomb repulsion of negative and positive DNA molecules respectively. This means that the concentration $N_{0}$ is located inside the window $N_{c} \leq N \leq N_{d}$ (see Fig. 1) and the width of the window grows with the strength of the short range attraction.

Below we present an analytical theory, which calculates both critical concentrations $N_{c}$ and $N_{d}$ in terms of the two main physical parameters of the system: boundary concentration of multivalent cations $N_{0}$ and the energy, $\varepsilon$, of the DNA binding within the DNA bundle per nucleotide.

At a small concentration of monovalent salt, $n$, the condensation threshold $N_{c}$ calculated in our theory is larger than the replacement concentration $N_{r}$. This means that the replacement of monovalent cations happens before the condensation and by itself can not lead to the con- densation while Coulomb forces are still strong. On the other hand, if $n$ is so large that $N_{r}$ is larger than the value of $N_{c}$ calculated in our theory (which assumes that replacement has happened), the condensation actually happens only at $N=N_{r}$. Thus, a large concentration of monovalent ions, $n$, acts as a "curtain". It eliminates a part of the window predicted by our theory. We show below that in the experiment of Ref. 3 the "curtain effect" starts to work only at $n>50 \mathrm{mM}$.

Two central parameters of our theory, $N_{0}$ and $\varepsilon$, can be calculated for a simple model of DNA as an uniformly charged cylinder 15 17. They can be also directly measured in independent experiments. The energy $\varepsilon$ was measured in Ref. 21.

On the other hand, one can obtain these parameters from the experimental values of $N_{c}$ and $N_{d}$. Below we use condensation and resolubilization for DNA with $\mathrm{Spe}^{4+}$ to estimate $\varepsilon$ and $N_{0}$. We obtain $\varepsilon=0.07 k_{B} T$ and $N_{0}=3.2 \mathrm{mM}$. The first number reasonably agrees with experimental data21.

Thus far we have talked about long DNA. For short DNA fragments, one should take into account the mixing entropy of DNA molecules which makes their solution more stable and makes the window between $N_{c}$ and $N_{d}$ smaller. We calculate the phase diagram for the condensation of DNA olygomers consisting of $L / b$ bases $(L$ is the length of olygomer, $b=1.7 \AA$ is length of double helix per phosphate). This phase diagram is shown in Fig. 2 on the plane $(N, y)$, where

$$
y=\frac{k_{B} T b}{\varepsilon L} \ln \frac{C_{\max }}{C},
$$

$C$ is the concentration of DNA molecules and $C_{\max }$ is its maximum value (equal to the inverse volume of a DNA molecule). Below the solid curve, in segregation domain, condensed and dissolved phases coexist. Thinking about very small concentrations of DNA, $C \ll C_{\max }$, we completely ignore the lower border of the segregation domain. Above the solid curve, all DNA molecules are in solution. A solution of long DNA molecules corresponds to a horizontal line $y=y_{0} \ll 1$. As concentration of DNA or its length decrease, the window between $N_{c}$ and $N_{d}$ shrinks. Condensation provides largest gain of free energy at $N=N_{0}$ where DNA molecules are neutral. This is why the phase boundary curve peaks at $N=N_{0}$. The dotted line on Fig. 2 which obeys equation $N=N_{0}$ devides the plane in two parts. At $N<N_{0}$ the net charge of a DNA molecule is negative, while at $N>N_{0}$ it is positive. This net charge by definition includes cations which are bound to DNA with binding enery larger than $k_{B} T$. Therefore, they move together with DNA in gel electrophoresis. This means that DNA should move in the direction opposite to the conventional one if $N>N_{0}$ 17. It would be interesting to verify this prediction experimentally. We propose also to combine experimental study of the condensation phase diagram with the gel electrophoretic measurement of the charge on the dissolved DNA molecules. 


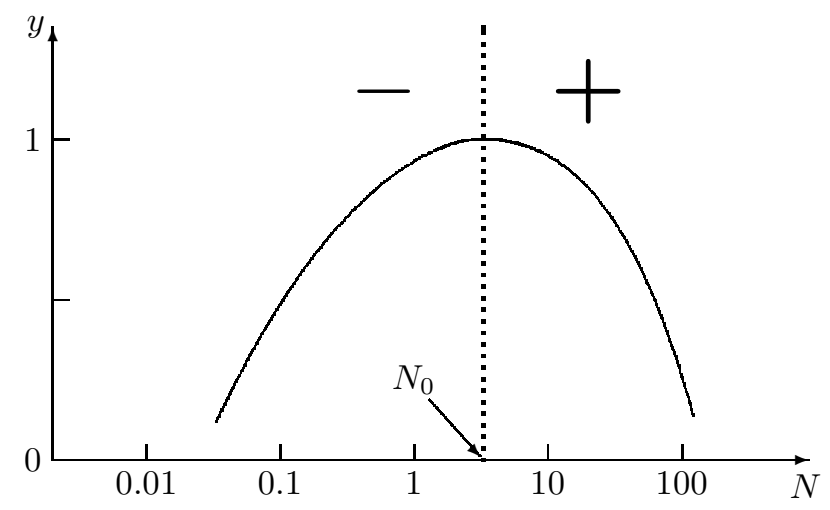

FIG. 2. Phase diagram of a dilute DNA solution in the plane of the bulk cation concentration $N$ (in units of $\mathrm{mM}$ ) and variable $y$ defined by Eq. (11). The solid curve was plotted using Eqs $(24)$ and 25 with fitting parameters obtained from the data 3 for long DNA screened by Spe ${ }^{4+}$. Above the curve, all DNA molecules are in solution $(x=1)$. Below the solid curve, the segregation domain is located. Here the condensed phase of DNA appears and in regions far enough from the curve, it consumes most of DNA $(x \ll 1)$. The dotted line corresponds to $N=N_{0}$, where the net charge of DNA changes sign from negative to positive

\section{CRITERION OF EQUILIBRIUM BETWEEN CONDENSED AND DISSOLLVED PHASES OF LONG DNA.}

In this section we consider long DNA molecules in an aqueous solution containing $Z$-valent counterions of the bulk concentration $N$. DNA condensation and resolubilization take place when the chemical potential of DNA in its condensed and dissolved states are equal, i.e.

$$
\mu_{c}=\mu_{d} \quad .
$$

For a long DNA we can neglect the entropy of DNA in both phases. We view the condensed phase as a bundle of parallel molecules which stick together due to correlation induced short range attraction to the nearest neigbours. The chemical potential of DNA molecule in the condensed phase in this approximation is determined by

$$
\mu_{c}=-\varepsilon L / b
$$

where $L / b$ is the number of negative charges in a DNA molecule.

Each molecule of a large bundle is practically neutral. More exactly its charge is inversly propotional to the number of molecules in the bundle 15 . This happens because the total charge of a large bundle keeps counterions inside the bundle very effectively.

On the other hand, in the dissolved state, each molecule aquires finite net charge density, $\eta^{*}$. This happens because a finite fraction of its counterions moves to the Debye-Hückel atmosphere in order to increase their entropy. One can view a single DNA molecule and its
Debye-Hückel atmosphere as a cylindrical capacitor with linear charge densities $\eta^{*}$ (inside) and $-\eta^{*}$ (outside). We recall that the net linear charge density of DNA, $\eta^{*}$, includes the bare charge and the charge of cations residing at the very surface of DNA and attached to the surface with binding energy larger than $k_{B} T$.

In equilibrium the electrostatic potential on the surface of DNA, $\varphi_{0}$, is determined by the ratio of the cation concentration in the bulk, $N$ and the concentration near the surface of DNA, $N_{0}$ :

$$
Z e \varphi_{0}=-k_{B} T \ln \frac{N_{0}}{N}
$$

This potential acts like a voltage difference applied to the capacitor.

The free energy per DNA molecule or the chemical potential of the dissolved phase, can be written as

$$
\mu_{d}=-\frac{1}{2} \varphi_{0} \eta^{*} L
$$

To derive Eq. (5) one should add the energy of the electric field of the capacitor,

$$
U=\frac{1}{2} \varphi_{0} \eta^{*} L
$$

to the change of the entropy term of the free energy of cations when they move from the surface of DNA to the bulk of solution

$$
\frac{L \eta^{*}}{Z e} k_{B} T \ln \frac{N_{0}}{N}=-\varphi_{0} \eta^{*} L
$$

(here Eq. (位) was used). Equivalent derivation can be found, for example, in Ref. 22. Comparing Eq. (5) and Eq. (6) we see that the change of the free energy of the capacitor is equal to the energy of an electric field with a minus sign. This is a realization of the general theorem valid for any capacitor kept at constant voltage 23 .

The surface potential, $\varphi_{0}$, can be easily related to the net charge density $\eta^{*}$. Indeed, at distance $r$ from its surface, a cylinder of radius $a$ and linear charge $\eta^{*}$ creates a potential

$$
\varphi(r)=\varphi_{0}-\frac{2 \eta^{*}}{D} \ln \left(\frac{a+r}{a}\right) .
$$

where $D$ is the dielectric constant of water. This potential vanishes beyond the Debye screening length,

$$
r_{s}=\left(4 \pi l_{B}\right)^{-1 / 2}\left(N Z^{2}+Z N+2 n\right)^{-1 / 2},
$$

where $l_{B}=e^{2} / D k_{B} T$ is the Bjerrum length. Therefore, substituting $r=r_{s}$ and $\varphi\left(r_{s}\right)=0$ into Eq. (8) one obtains

$$
\varphi_{0}=\frac{2 \eta^{*}}{D} \ln \left(\frac{a+r_{s}}{a}\right) .
$$


Substituting Eq. (10) into Eq. (5) we get

$$
\mu_{d}=-\frac{\left(\eta^{*}\right)^{2}}{D} L \ln \left(1+r_{s} / a\right)
$$

Using the condition (2) together with the expressions for the chemical potentials for both states, Eqs. (3) and (11) we arrive at the final equation for the DNA reentrant condensation transitions

$$
\frac{\varepsilon}{k_{B} T}=\frac{b\left(\eta^{*}\right)^{2}}{D} \ln \left(1+r_{s} / a\right)
$$

To proceed further one has to know the net charge density, $\eta^{*}$. Next section gives a review of current understanding of this quantity.

\section{NET LINEAR CHARGE DENSITY OF SCREENED DNA}

Conventional understanding of nonlinear screening of a strongly charged cylinder is based on the PoissonBoltzmann equation. Let us first consider a cylinder with a negative bare linear charge density, $-\eta$, which is screened by $Z$-valent cations $(n=0)$. Assume that $\eta>\eta_{c}$, where $\eta_{c}=e / l_{B}\left(\eta=4.2 \eta_{c}\right.$ for double helix DNA). Onsager and Manning 18 argued that such a cylinder is partially screened by counterions residing at the very surface, so that the net linear charge density of the cylinder, $\eta^{*}$, is equal to the negative universal value $-\eta_{c} / Z$. The rest of the cylinder charge is linearly screened at much larger distances according to the linear Debye-Hückel theory. The net charge, $\eta^{*}$, does not depend on the bulk concentration of cations, $N$ and is shown (in units of $-\eta_{c}$ ) by the dotted line on Fig. 3a. The Onsager-Manning picture of condensation was confirmed by the solution of the Poisson-Boltzmann equation 19 .

Let us now discuss a water solution, containing both a concentration, $n$, of monovalent salt and a concentration, $N$ of $Z: 1$, salt. When $N$ grows to some well defined concentration, $N=N_{r}$, multivalent cations replace monovalent ones at the surfage of DNA. According to conventional understanding 2425 this replacement of the condensed monovalent ions changes $-\eta^{*} / \eta_{c}$ from 1 to $1 / Z$ (see the dotted line on Fig. 3b). In logarithmic scale this transition_logks quite abrupt.

In recent paper $\$ 1,17$ the influence of strong correlations of multivalent cations at the surface of a macroion on its net charge, $\eta^{*}$, was studied. The general expression for the net linear charge density, $\eta^{*}$, was derived:

$$
\eta^{*}=-\frac{\eta_{c}}{2 Z} \frac{\ln \left(N_{0} / N\right)}{\ln \left(1+r_{s} / a\right)}
$$

This equation takes into account correlations with the help of the new boundary condition $N(r=0)=N_{0}$ for the Poisson-Boltzmann equation. To remind derivation of this result let us write down the Boltzmann formula

$$
N(r)=N_{0} \exp \left(-\frac{Z e}{k_{B} T}\left(\varphi(r)-\varphi_{0}\right)\right)
$$

At $r=r_{s}$ the concentration $N(r)$ reaches its bulk value, $N$. Substituting Eq. (10) into Eq. (14) we obtain

$$
N=N_{0} \exp \left(\frac{2 Z \eta^{*}}{\eta_{c}} \ln \left(\frac{a+r_{s}}{a}\right)\right) .
$$

Expressing $\eta *$ in terms of the bulk cation concentration $N$ we arrive at Eq. (13). This equation was used to plot solid lines on Fig. 3.
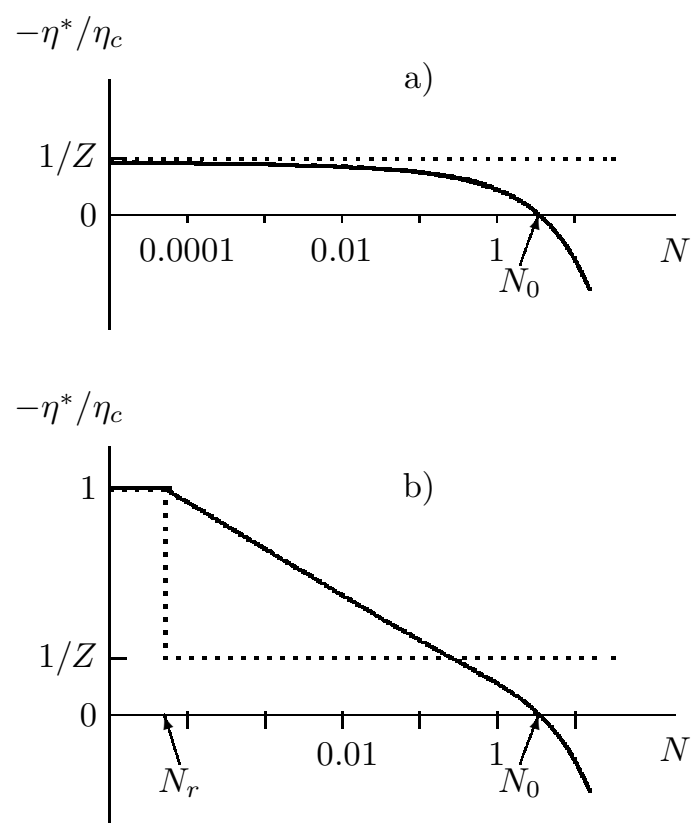

FIG. 3. The dimensionless net linear charge density $\eta^{*} / \eta_{c}$ as function of $Z$-valent cation concentration $N$ (in units of $\mathrm{mM}$ ) at zero concentration $n$ of monovalent salt (a) and at finite $n$ (b). The solid curves are drawn according Eq. (13) with parameters obtained from the data 3 for DNA screened by $\mathrm{Spe}^{4+}$. Dotted curves represent conventional understanding of results of Poisson-Bolzmann equation. $N_{0}$ is the concentration of $Z$-valent ions at the surface of DNA and $N_{r}$ is the concentration $N$ at which $Z$-valent cations replace monovalent ones.

At $n=0$ (Fig. 3a) one can seet that Manning's limiting value of $\eta^{*}=-\eta_{c} / Z$ holds only for the unrealistically small $N$. At larger $N$ the absolute value of the net charge decreases, $\eta^{*}$ crosses zero and becomes positive.

At finite $n$, the screening radius, $r_{s}$, strongly decreases and at small $N$ the net charge density, $-\eta^{*}$, becomes larger than $\eta_{c} / Z$ (see solid line on Fig. 3b). It was shown in Ref. 17 that when $N$ goes to $N_{r}$, the density $\eta^{*}$ becomes as large as $\eta_{c}$, so that at the replacement point the net density, $\eta^{*}$, matches its standard value for monovalent ions. At larger $N$ the role of the monovalent salt is smaller and $\eta^{*}$ is similar to that of $n=0$. The density, $\eta^{*}$, crosses zero at $N=N_{0}$ and becomes positive. In the 
next section we will use Eq. (13) to find the condensation and resolubilization thresholds.

\section{REENTRANT CONDENSATION THRESHOLDS FOR LONG DNA MOLECULES. COMPARISON WITH EXPERIMENT}

Now we can substitute Eq. (13) into Eq. (12) and obtain an explicit equation for the threshold concentrations:

$$
\frac{\varepsilon}{k_{B} T}=\frac{1}{4 Z^{2} \xi} \frac{\ln ^{2}\left(N_{0} / N\right)}{\ln \left(1+r_{s} / a\right)}
$$

Here $\xi=l_{B} / b$ is the conventional Manning's parameter $(\xi=4.2$ for DNA). Obviously, there exist two solutions, $N_{c}$ and $N_{d}$, for the bulk concentration, $N$, which corresponds respectively to the condensation and dissolution transition points.

Concentration $N_{c}$ is usually so small that $r_{s}$ is dominated by monovalent ions and $r_{s}=r_{1}=\left(8 \pi l_{B} n\right)^{-1 / 2}$. With the help of Eq. (16) we arrive at a simple equation for $N_{c}$ :

$$
\frac{\varepsilon}{k_{B} T}=\frac{1}{4 Z^{2} \xi} \frac{\ln ^{2}\left(N_{0} / N_{c}\right)}{\ln \left(1+r_{1} / a\right)}
$$

To obtain an equation for $N_{d}$, we take into account that in the case of $\mathrm{Spe}^{4+}$ cation 3 , the condensed phase dissolves at $N_{d} \approx 150 \mathrm{mM}$. At such a large concentration, $r_{s}$ is determined by multivalent cations. However, if we use Eq. (9), $r_{s}$ turns out to be much smaller than the average distance between cations in the bulk of solution, which does not make physical sense. This means that the Debye-Hückel theory does not work. A natural approximation in this case is to replace $r_{s}$ by average distance to closest cation $r_{d}=\left(4 \pi N_{d} / 3\right)^{-1 / 3}$. This gives for $N_{d}$

$$
\frac{\varepsilon}{k_{B} T}=\frac{1}{4 Z^{2} \xi} \frac{\ln ^{2}\left(N_{0} / N_{d}\right)}{\ln \left(1+r_{d} / a\right)}
$$

In practice, the concentrations $N_{c}$ and $N_{d}$ are known from experiments, so that important parameters $\varepsilon$ and $N_{0}$ can be found with the help of Eqs. (17) and (18). For example, $N_{0}$ can be found by eliminating $\varepsilon$ from eqs.(17, 18). In the specific experimental situation of Ref. 3 where long DNA molecules condensed with a 4valent cation, $\mathrm{Spe}^{4+}, N_{c}=0.025 \mathrm{mM}$ and $N_{d}=150 \mathrm{mM}$ at the lowest concentration $n=10 \mathrm{mM}$ of $\mathrm{NaCl}$. Using these values we obtain from Eqs. (17), (18) $N_{0}=3.2 \mathrm{mM}$ and $\varepsilon / k_{B} T \approx 0.07$. The last value favorably agrees with the energy of attraction between the CoHex condensed DNA obtained in the osmotic stress experiment 21 $\varepsilon=0.08 k_{B} T$.

Knowing $N_{0}$ and $\varepsilon$, we can try to reproduce the experimental dependence of the condensation threshold $N_{c}$ on concentration of $\mathrm{NaCl}$, obtained in Ref. 3. This can be done with the help of Eq. (17):

$$
\ln N_{c}=\ln N_{0}-\left(4 Z^{2} \xi \frac{\varepsilon}{k_{B} T} \ln \left(1+r_{1} / a\right)\right)^{1 / 2}
$$

The calculated function $N_{c}(n)$ is shown on Fig. 4 together with the experimental points from Ref. 3. It is clear that Eq. (19) closely reproduces experimental behavior till $n \lesssim 50 \mathrm{mM}$. The later value is close to the concentration of monovalent cations which is needed to replace 4 -valent cations at the surface of DNA for $N \approx 0.01 \mathrm{mM}$. It seems that at $n>50 \mathrm{mM}$, condensation happens simultaneously with the replacement of monovalent cations by multivalent ones, i.e. at $N_{c} \sim N_{r}$, as was assumed in Ref. 25.

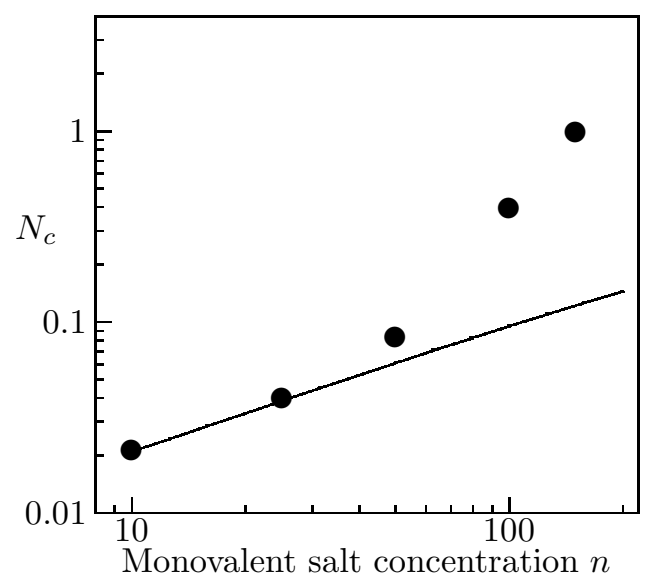

FIG. 4. The condensation threshold $N_{c}$ as a function of monovalent salt concentration, $\%$. The solid curve corresponds to Eq. (19). Experimental data 3 are shown by the large dots.

We would like to emphasize that in the broad range of lower monovalent salt concentrations $n<50 \mathrm{mM}$ when multivalent cations cover the DNA surface, condensation does not happen. In this range, the Coulomb repulsion of negative DNA molecules is strong enough to prevent condensation because, as shown on Fig. 3b, the absolute value of the net negative charge on the polymer, $-\eta^{*}$, does not abrubtly decrease from $\eta_{c}$ to $\eta_{c} / Z$ at $N_{c} \sim N_{r}$, but still stays larger than $\eta_{c} / Z$ in the large interval of $N>N_{r}$.

In contrast with $N_{c}$, the resolubilization threshold $N_{d}$ practically does not depend on $n$. This happens because at $N=N_{d}$, screening is dominated by multivalent cations and agrees with our Eq. (18).

Let us now discuss another experimental studyt in which DNA were condensed with trivalent $\mathrm{Spd}^{3+}$ cations. In this case, for $n=4 \mathrm{mM}$ thresholds are equal $N_{c} \approx$ $2 \mathrm{mM}$ and $N_{d} \approx 60 \mathrm{mM}$, which yields $N_{0} \approx 11 \mathrm{mM}$ and $\varepsilon / k_{B} T \approx 0.02$. This value of $\varepsilon / k_{B} T$ is about three times smaller than for $\mathrm{Spe}^{4+}$. This is quite reasonable, if attraction has an electrostatic nature.

The concentrations, $N_{0}$, we obtained above are substantially larger than the microscopic theory 17 predicts for the point-like 3- and 4-valent cations. Actually, 
cations $\mathrm{Spd}^{3+}$ and $\mathrm{Spe}^{4+}$ are quite long $(\approx 15$ and $20 \AA$ respectively) linear polymers. Their length is approximately equal to the average distance between cation centers in two-dimensional strongly correlated liquid of cations on the surface of DNA. Thus, to obtain a more reliable theoretical prediction of $N_{0}$, one has to study the thermodynamic properties of the strongly correlated (possibly nematic) liquid of these ions on an uniform negative background by numerical methods. We will address this problem in our next paper.

The application of our theory to experimental datalis is not completely convincing, because of the following. Our theory assumes that the concentration of DNA is so small that the concentration of DNA phosphates $N_{p h}$ is smaller than $N$, so that the DNA charge can always be compensated bu Z-valent cations. Actually in experimental conditions 3. the condensation threshold $N_{c}$ happens to be close to $N_{p h}$. It is possible, therefore, that $N_{c}$ in this case is determined by the condition $N_{c} \sim N_{p h}$ rather than the repulsion of DNA helices. This means that the actual value of $N_{c}$ can be lower than the measured one. Therefore, the true values of $N_{0}$ should be somewhat smaller, and that of $\varepsilon$ should be somewhat larger than our estimates. We suggest a repetition of experiments at smaller concentrations of long DNA.

Another way to improve the reliability of the extraction of the value of $N_{0}$ from experiment is to study the condensation of short DNA (see next section).

Note that Ref. 3 also contains information on the single and triple stranded DNA helices, which condensed in the narrower and wider range of $\left[\mathrm{Spe}^{4+}\right]$ than the double helices respectively. This tendency agrees with idea that correlations play the major role in this phenomenon.

\section{CONDENSATION OF SHORT DNA MOLECULES.}

In this Section we explicitly deal with the mixing entropy of DNA molecules in the dissolved state, and calculate the phase diagram for the DNA solution. We consider double helix DNA molecules of length $L$, with $L / b$ bases each.

The free energy per DNA molecule in solution with concentration $C$ is

$$
F=-x k_{B} T\left(\ln \left(\frac{C_{\max }}{C x}\right)+1\right)-(1-x) \frac{L}{b} \Delta,
$$

where $1-x$ is the fraction of DNA molecules in the condensate. The first term in Eq. (20) is the entropy of the dissolved DNA phase per molecule of DNA. Here $C_{\max } \sim 1 / \pi a^{2} L$ is the inverse volume of the DNA molecule. The second term in Eq. (20) is the average free energy per molecule in the condensed state. Here $\Delta$ is the difference between the energy of short range attraction $\varepsilon$ and the free energy $b \mu_{d} / L$ of the screening atmosphere per nucleotide (see Eq. (11)):

$$
\frac{\Delta}{k_{B} T}=\frac{\varepsilon}{k_{B} T}-\frac{1}{4 Z^{2} \xi} \frac{\ln ^{2}\left(N_{0} / N\right)}{\ln \left(1+r_{s} / a\right)} .
$$

Minimizing Eq. (20) with respect of $x$, we find

$$
x=\frac{C_{\max }}{C} \exp \left(-\frac{\Delta}{k_{B} T} \frac{L}{b}\right) .
$$

Then the boundary of two-phase domain $x=1$ corresponds to the condition

$$
\ln \frac{C_{\max }}{C}=\frac{\Delta}{k_{B} T} \frac{L}{b}
$$

which can be explicitly written for $N<N_{0}$ as

$$
\frac{b k_{B} T}{L \varepsilon} \ln \frac{C_{\max }}{C}=1-\frac{k_{B} T}{\varepsilon} \frac{1}{4 Z^{2} \xi} \frac{\ln ^{2}\left(N_{0} / N\right)}{\ln \left(1+r_{1} / a\right)},
$$

and for $N>N_{0}$

$$
\frac{b k_{B} T}{L \varepsilon} \ln \frac{C_{\max }}{C}=1-\frac{k_{B} T}{\varepsilon} \frac{1}{4 Z^{2} \xi} \frac{\ln ^{2}\left(N_{0} / N\right)}{\ln \left(1+r_{N} / a\right)} .
$$

where $r_{N}=(4 \pi N / 3)^{-1 / 3}$. The last three equations specify the conditions when the chemical potentials of the condensed and dissolved states of DNA are equal. The curve described by Eqs. (24) and (25), forms a boundary on the phase diagram, separating the region of the dissolved DNA (above it) from the region where the condensed and dissolved phases of DNA coexist (below it). Within the separation domain the fraction of dissolved DNA is given by Eq. (22).

The position and the shape of the phase boundary obviously depends on the values of the parameters $N_{0}$, $\varepsilon / k_{B} T$ and $2 Z^{2} \xi$. For double-helix DNA with $\mathrm{Spe}^{4+}$ we find $2 Z^{2} \xi=134$ and using the estimates $\varepsilon / k_{B} T=0.07$ and $N_{0} \approx 3.2 \mathrm{mM}$ obtained above we can calculate the phase boundary for condensation of the DNA fragments of the arbitrary length. This boundary is shown on Fig. 2 by the solid line.

The dotted line $N=N_{0}$ divides the phase diagram of Fig. 2 into two regions where the DNA molecules have opposite signs. Positive net charge of dissolved DNA can be measured in an electrophoresis experiment because cations included in this charge are bound to DNA with a binding energy larger than $k_{B} T$ and therefore move together with DNA molecule. At $N>N_{0}$ one should see that DNA molecules are positive both above and below phase boundary of Fig. 2. However, below the boundary, intensity of corresponding electrophoresis peak should decay rapidly with the distance from the boundary. This intensity is picked up by slowly moving bundles of DNA molecules, which at large enough $N$ can be also positive. 


\section{CONCLUSION}

The theory of DNA condensation and resolubilization by multivalent cations presented above makes several novel, well-defined predictions which have not been confirmed by experiment yet. The main results of the study are summarized in the phase diagram presented on Fig. 2. There are only two physical parameters $\varepsilon$ and $N_{0}$ on which the shape of the phase boundary depends. Therefore measuring just a few threshold concentrations of multivalent cations $N_{c}$ and $N_{d}$ for solution of DNA fragments of different length and/or concentrations should yield several independent determinations of these quantities, and at the same time provide the test for the selfconsistency of our model. Experimental studies of DNA phase diargam with different multivalent cations would provide the values of the attractive energy for different ions. In the present theory the origin of parameters $\varepsilon$ and $N_{0}$ was not specified and they were treated as phenomenological parameters. Comparison of experimentally determined $\varepsilon$ and $N_{0}$ values for different counterions should yied information about the nature of the attraction. It is worth noting here that if the attraction is of electrostatic correlation origin the value of $\varepsilon$ in principle includes attraction due to the correlations of multivalent cations as well as all of the repulsive non-Coulomb DNADNA interaction. However, the first interaction decays slower than the second one. Then the binding energy $\varepsilon$ is determined by correlations. In this case, the two quantities $\varepsilon$ and $N_{0}$ are not independent 1517 namely $\ln N_{0} \propto \varepsilon$. For the model of the uniformly charged cylinder both parameters were calculated microscopically 15.17.

We would like to emphasize that the concentration $N_{0}$ found in this paper plays extremely important role in any phenomenon related to screening of DNA molecules by multivalent ions 16.17 . In this paper, we try to attract attention to the fact that $N_{0}$ plays major role in electrophoresis, because the net linear charge density of DNA $\eta^{*}$ changes sign at $N=N_{0}$. It was predicted 16,17 that DNA should start moving in the opposite direction at $N>N_{0}$. It is not obvious that one can see this phenomenon for long DNA. Indeed, in large interval of concentrations $N_{0}<N<N_{d}$ most of long DNA molecules are condensed in low mobilty bundles while concentrations $N>N_{d}$ may be difficult for experiment because of large dissipation of heat. Therefore, we suggest doing electrophoresis of a solution of short DNA fragments. In this case all DNA molecules have unconventional sign of mobility at smaller than $N_{d}$ concentrations. The phase diagram shown in Fig. 2 predicts good conditions for such an experiment. It would be very interesting to verify predicted correlations between the reentrant condensation and unconventional electrophoresis.

\section{ACKNOWLEDGMENTS}

We are grateful to A. Yu. Grosberg, V. A. Bloomfield and R. Podgornik for valuable discussions. This work was supported by NSF DMR-9616880 (T. N. and B.S) and NIH GM 28093 (I. R.).

${ }^{1}$ V. A. Bloomfield, Curr. Opinion Struct. Biol.6, 334 (1996).

${ }^{2}$ V. A. Bloomfield, Biopolymers. 44, 269 (1998).

${ }^{3}$ M. Saminathan, T. Antony, A. Shirahata, L. Sigal, T. Thomas and T. J. Thomas, Biochemistry. 38, 3821 (1999).

${ }^{4}$ J. Pelta, D. Durand, J. Doucet and F. Livolant, Biophysical Journal. 71, 48 (1996).

${ }^{5}$ J. Pelta, F. Livolant and J.-L. Sikorav, Journal of Biological Chemistry. 271, 5656 (1996).

${ }^{6}$ E. Raspaud, M. Olvera de la Cruz, J.-L. Sikorav and F. Livolant, Biophysical Journal. 74, 3811998.

${ }^{7}$ F. Oosawa, Biopolymers. 6, 134 (1968).

${ }^{8}$ L. G. Gulbrand, Bo Jonsson, H. Innerstrom, and P. Linse, J. Chem. Phys. 80, 2221(1984).

${ }^{9}$ R. Kjellander, Ber. Bunsenges. Phys. Chem. 100, 894 (1996).

${ }^{10}$ I. Rouzina and V. A. Bloomfield, J. Phys. Chem. 100, 9977, (1996).

${ }^{11}$ N. Gronbech-Jensen, R. J. Mashl, R. F. Bruinsma, and W. M. Gelbart, Phys. Rev. Lett. 78, 2477 (1997).

12 B. J. Ha and A. J. Liu, Phys. Rev. Lett. 79, 1289 (1997); Phys. Rev. Lett. 81, 1011 (1998).

${ }^{13}$ R. Podgornik and V. A. Parsegian, Phys. Rev. Lett. 80, 1560 (1998).

${ }^{14}$ J. J. Arenzon, J. F. Stilck, and Y. Levin, condmat/9806358

15 B. I. Shklovskii, Phys. Rev. Lett. 82, 3268 (1999).

${ }^{16}$ V. I. Perel and B. I. Shklovskii, cond-mat/9902016, Physica A, (1999)

${ }^{17}$ B. I. Shklovskii, cond-mat/9907351; Phys. Rev. E. (1999).

${ }^{18}$ G. S. Manning, J. Chem. Phys. 51, 924 (1969).

19 B. Zimm, M. LeBret J. Biomol. Struct. Dyn. 1, 461(1983).

${ }^{20}$ S. Leikin, A. A. Kornyshev, Phys. Rev. Lett. 82, 4138 (1999).

${ }^{21}$ D. Rau and A. Parsegian, Biophysical Journal. 61, 246 (1992).

22 S. A. Safran, Statistical Thermodynamics of surfaces, interfaces and membranes 1994, Addison-Wesley.

${ }^{23}$ L. D. Landau and E. M. Lifshitz, Electrodynamics of continuous media, Chapter 1, Oxford, Pergamon Press (1998).

${ }^{24}$ G. S. Manning, Biophys. Chem. 7, 95 (1999).

${ }^{25}$ R. W. Wilson, V. A. Bloomfield, Biochemistry. 18, 2192 (1999). 